Portland State University

PDXScholar

Electrical and Computer Engineering Faculty

Publications and Presentations

10-1-1981

\title{
Modes of a laser resonator with a retroreflecting roof mirror
}

Guosheng Zhou

Lee W. Casperson

Portland State University

Follow this and additional works at: https://pdxscholar.library.pdx.edu/ece_fac

Part of the Electrical and Computer Engineering Commons

Let us know how access to this document benefits you.

\section{Citation Details}

Guosheng Zhou, Lee W. Casperson. Modes of a laser resonator with a retroreflecting roof mirror. Applied Optics, Volume 20, Number 20 (October 1981), pp. 3542-3546.

This Article is brought to you for free and open access. It has been accepted for inclusion in Electrical and Computer Engineering Faculty Publications and Presentations by an authorized administrator of PDXScholar. Please contact us if we can make this document more accessible: pdxscholar@pdx.edu. 


\title{
Modes of a laser resonator with a retroreflecting roof mirror
}

\author{
Guosheng Zhou and Lee W. Casperson
}

\begin{abstract}
The self-consistent integral equation for the field distribution of the resonant modes in a resonator with a tilted retroreflecting roof mirror is solved. The field distribution in the direction of the roof can be described in terms of Hermite-Gaussian functions. The beam matrix for a retroreflecting roof is found, and a new type of resonator which does not need precise alignment is proposed.
\end{abstract}

\section{Introduction}

The flat-roof laser resonator has been investigated by several authors. Di Francia's ${ }^{1}$ analysis was based on the (closed) diamond-shaped cavity, $\mathrm{Kahn}^{2}$ solved this problem by ray theory, while Checcacci et al. evaluated the Fresnel integral equation using an iterative Fox and Li method. ${ }^{3-5}$ Experimental investigations have been carried out at microwave frequencies. ${ }^{4,5}$ All these studies have dealt with only the flat-roof resonator, in which the angles of the roofs are extremely small (of the order of $10^{-3} \mathrm{rad}$ ) and the flat roof is perpendicular to the optical axis. Bergstein et al. ${ }^{6}$ proposed a symmetric resonator with two right-angle roof mirrors, but mathematical details were not included.

The important advantage of the roof mirror in laser resonators is that within certain approximations it returns a bundle of rays parallel to its original path. Thus, in some respects the device acts as a flat mirror perpendicular to the beam regardless of the rotational angle around the roof line. In principle, at least, the resulting resonators should be relatively insensitive to misalignment in one direction. For this reason the retroreflecting roof has been used to terminate ruby rodes, and rotating roof mirrors have been used as laser Qswitches. However, a detailed theoretical analysis of lasers with retroreflecting roof mirrors has not yet been given. In particular, it is not known what the effect

The authors are with University of California at Los Angeles, School of Engineering \& Applied Science, Los Angeles, California 90024.

Received 18 April 1981.

$0003-6935 / 81 / 203542-05 \$ 00.50 / 0$.

(C) 1981 Optical Society of America. might be of the transverse ray displacement at the roof, and the amplitude and phase distributions of the electromagnetic modes are unknown.

This paper presents a detailed diffraction integral calculation of the modes of a retroreflecting roof resonator with a roof angle of $90^{\circ}$ and arbitrary roof misalignments. Surprisingly, for an appropriate choice of coordinates the ray properties of a retroreflecting roof mirror can be described with a simple unimodular $2 \times$ 2 ray matrix. The modes of a resonator consisting of a roof mirror facing a spherical mirror are almost the same as the Hermite-Gaussian distributions obtained in conventional spherical mirror resonators. One important difference is that an additional mode dependent phase shift occurs in lasers with retroreflecting mirrors. Thus the mode frequencies of lasers with retroreflecting mirrors are different from the frequencies of conventional resonators, even though the amplitude distributions are similar.

Starting from the results given here one could estimate the mode distortions that would occur for excessive misalignment of a retroreflecting resonator mirror or imperfections in the mirror surfaces. The stability of these modes with respect to perturbations in the resonator can also be determined. In regard to ease of fabrication, the right-angle roof reflectors are probably less convenient than spherical mirrors in the optical region, but the resulting insensitivity to misalignment may be an important advantage. These mirrors can also compensate for the distortion caused by a prism, and they might be useful for compensating 1-D distortion in chemical lasers or large volume gas lasers.

The basic self-consistent integral equation is derived in Sec. II, and the solutions for resonators with retroreflecting roof and spherical mirrors are given in Sec. III. The ray matrix for a retroreflecting roof is given in Sec. IV, and a resonator consisting of two retroreflecting roof mirrors and a cylindrical lens is also described. The advantage of this resonator is that it supports simple Hermite-Gaussian modes and is extremely insensitive to angular alignment. 


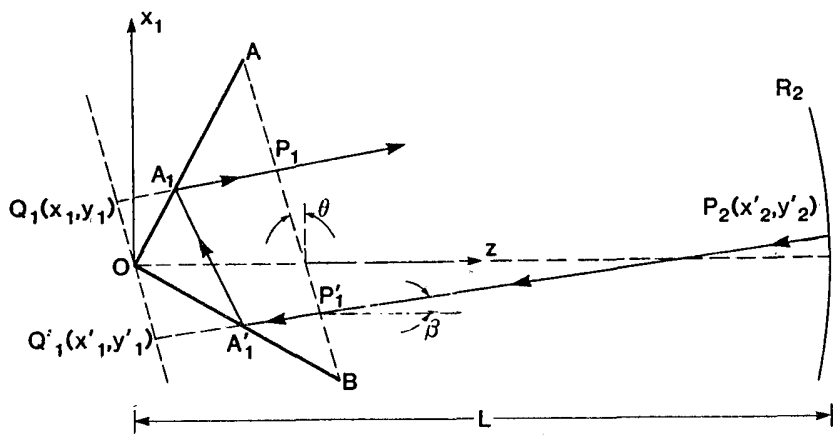

Fig. 1. Schematic representation of a light ray in a resonator with a retroreflecting roof.

\section{Self-Consistent Integral Equation}

The retroreflecting roof resonator is represented in Fig. 1. It consists of a roof mirror facing a spherical mirror. The reflectivities of both roof and spherical mirror are assumed to be uniform. The spherical mirror has a radius of curvature $R_{2}$ and is square in cross section. The optical axis ( $z$ axis) passes through the center of the spherical mirror and the apex of the retroreflecting roof mirror $O$ and is perpendicular to the roof line. The $y$ axis is parallel to the roof line. The coordinate origin is the apex of the roof, and the distance between the center of the spherical mirror and the apex $O$ is represented by $L$. The angle between the $x$ axis and the retroreflective roof surface is $\theta$, which is positive when the surface of the roof is turned counterclockwise from the $x$ axis.

An incident ray from the point $P_{2}\left(x_{2}, y_{2}\right)$ passes through the point $P_{1}^{\prime}$ on the surface of the roof mirror and impinges at the point $A_{1}^{\prime}$. This ray then experiences two reflections at $A_{1}^{\prime}$ and $A_{1}$ and goes to $P_{1}$ at the surface of the roof mirror. The reflected ray is parallel to the incident ray $P_{2} P_{1}^{\prime}$. It is shown in the Appendix that the path length difference $\Delta$ from the point $P_{1}^{\prime}$ through $A_{1}^{\prime}$ and $A_{1}$ to $P_{1}$ can be written in the form

$$
\Delta=d \sec \theta-d \tan \theta \sec \theta\left(x_{1}+x_{2}\right) / L,
$$

where $d=A B$ is the width of the roof. The path length difference from the point $P_{2}\left(x_{2}, y_{2}\right)$ through $P_{1}^{\prime}$ and $A_{1}^{\prime}$ to $Q_{1}^{\prime}\left(\dot{x}_{1}^{\prime} y_{1}^{\prime}\right)$ can be expressed in the form ${ }^{7}$

where

$$
\rho^{\prime}\left(x_{1}^{\prime}, y_{1}^{\prime} ; x_{2}, y_{2}\right)=\rho^{\prime}\left(x_{1}^{\prime}, x_{2}\right)+\rho\left(y_{1}^{\prime}, y_{2}\right),
$$

$$
\begin{aligned}
\rho^{\prime}\left(x_{1}^{\prime}, x_{2}\right) & \simeq L / 2+x_{1}^{\prime} \tan \theta+\left(x_{1}^{\prime 2}+g_{2} x_{2}^{2}-2 x_{1}^{\prime} x_{2}\right) / 2 L, \\
\rho\left(y_{1}^{\prime}, y_{2}\right) & \cong L / 2+\left(y_{1}^{\prime 2}+g_{2} y_{2}^{2}-2 y_{1}^{\prime} y_{2}\right) / 2 L, \\
g_{2} & =1-L / R_{2} .
\end{aligned}
$$

The path length difference from the point $Q_{1}\left(x_{1}, y_{1}\right)$ to $P_{2}\left(x_{2}, y_{2}\right)$ is given by

$$
\rho\left(x_{1}, y_{1} ; x_{2}, y_{2}\right)=\rho^{\prime \prime}\left(x_{1}, x_{2}\right)+\rho\left(y_{1}, y_{2}\right),
$$

where

$$
\rho^{\prime \prime}\left(x_{1}, x_{2}\right)=L / 2+x_{1} \tan \theta+\left(x_{1}^{2}+g_{2}^{2} x_{2}^{2}-2 x_{1} x_{2}\right) / 2 L .
$$

The self-consistent Fresnel-Kịchhoff integral equation can be expressed as follows:

$$
\begin{aligned}
\gamma E^{(2)}\left(x_{2}, y_{2}\right)= & \iint d x_{2}^{\prime \prime} d y_{2}^{\prime \prime} K\left(x_{2}^{\prime \prime}, y_{2}^{\prime \prime} ; x_{2}, y_{2}\right) E^{(2)}\left(x_{2}^{\prime \prime}, y_{2}^{\prime \prime}\right), \\
K\left(x_{2}^{\prime \prime}, y_{2}^{\prime \prime} ; x_{2}, y_{2}\right)= & (i / \lambda)^{2} \iint d x_{1}^{\prime \prime} d y_{1}^{\prime \prime} \exp \left\{\left[-i k \rho^{\prime}\left(-x_{1}^{\prime \prime}, y_{1}^{\prime \prime} ; x_{2}^{\prime \prime}, y_{2}^{\prime \prime}\right)\right.\right. \\
& \left.\left.-i k \rho\left(x_{1}^{\prime \prime}, y_{1}^{\prime \prime} ; x_{2}, y_{2}\right)\right]\right\}\left[\rho^{\prime}\left(-x_{1}^{\prime \prime}, y_{1}^{\prime \prime} ; x_{2}^{\prime \prime}, y_{2}^{\prime \prime}\right)\right. \\
& \left.+\rho\left(x_{1}^{\prime \prime}, y_{1}^{\prime \prime} ; x_{2}, y_{2}\right)\right]^{-1},
\end{aligned}
$$

where the double primes denote integration variables corresponding to $x_{1}, y_{1}, x_{2}$, and $y_{2}$. The field on the surface of the spherical mirror is represented by $E^{(2)}$ $\left(x_{2}, y_{2}\right)$ and $\gamma$ is the corresponding eigenvalue. $K$ is the kernel of the integral equation. The wave number is $k=2 \pi / \lambda$, and $\lambda$ is wavelength in the resonator. Here the relationship $x_{1}=-x_{1}^{\prime}$ has been used (see Appendix).

Since the kernel of the integral equation $K\left(x_{2}^{\prime \prime}, y_{2}^{\prime \prime} ; x_{2}, y_{2}\right)$ can be separated in the $x$ and $y$ directions, the field expression splits into two independent integral equations, one for each lateral coordinate. These can be written as follows (see Appendix):

$$
\begin{aligned}
\gamma_{x} E_{x}^{(2)}\left(x_{2}\right)= & (i / \lambda L) \int d x_{2}^{\prime \prime} E_{x}^{(2)}\left(x_{2}^{\prime \prime}\right) \int d x_{1}^{\prime \prime}\left(1-x_{1}^{\prime \prime} \tan \theta / L\right)^{-1} \\
& \cdot\left(1+x_{1}^{\prime \prime} \tan \theta / L\right)^{-1} \exp \left\{-i k\left[\rho^{\prime}\left(-x_{1}^{\prime \prime}, x_{2}^{\prime \prime}\right)\right.\right. \\
& \left.\left.+\rho^{\prime \prime}\left(x_{1}^{\prime \prime}, x_{2}\right)\right]\right\} \\
\gamma_{y} E_{y}^{(2)}\left(y_{2}\right)= & (i / \lambda L) \int d y_{2}^{\prime \prime} E_{y}^{(2)}\left(y_{2}^{\prime \prime}\right) \\
& \cdot \int d y_{1}^{\prime \prime} \exp \left\{-i k\left[\rho\left(y_{1}^{\prime \prime}, y_{2}^{\prime \prime}\right)+\rho\left(y_{1}^{\prime \prime}, y_{2}\right)\right]\right\}, \\
E^{(2)}\left(x_{2}, y_{2}\right)= & E_{x}^{(2)}\left(x_{2}\right) E_{y}^{(2)}\left(y_{2}\right), \\
\gamma= & \gamma_{x} \gamma_{y},
\end{aligned}
$$

where $E_{x}^{(2)}\left(x_{2}\right)$ and $E_{y}^{(2)}\left(y_{2}\right)$ illustrate; respectively, the field variations in the $x$ and $y$ directions on the surface of the spherical mirror, with $\gamma_{x}$ and $\gamma_{y}$ as the corresponding eigenvalues. According to the approximate rules used in the Huygens-Kirchhoff equation, in the integrand function small quantities to first order are kept and in the exponential small quantities to second order are kept. The factor $\left(1-x_{1}^{\prime \prime} \tan \theta / L\right)\left(1+x_{1}^{\prime \prime}\right.$ $\tan \theta / L)$ comes from the distance $P_{1} P_{2}$ in the denominator. The limits of the second integration with respect to $x_{1}^{\prime \prime}$ and $y_{1}^{\prime \prime}$ are assumed to approach infinity, which can be interpreted to mean that the modes of the resonator will be sufficiently confined about the axis and the roof mirror be sufficiently large that the contribution to the integral from points other than those close to the axis may be neglected.

By substituting Eqs. (3) and (7) into Eq. (10) and integrating with respect to $x_{1}^{\prime \prime}$, we can simplify Eq. (10) as follows:

$$
\begin{aligned}
\gamma_{x} E_{x}^{(2)}\left(x_{2}\right)= & \exp (i \pi / 4-i k L)(2 \lambda L)^{-1 / 2} \\
& \cdot \int_{-\infty}^{\infty} d x_{2}^{\prime \prime} E_{x}^{(2)}\left(x_{2}^{\prime \prime}\right) \exp \left\{-\frac{i k}{4 L}\left[\left(2 g_{2}-1\right)\left(x_{2}^{2}+x_{2}^{\prime \prime 2}\right)\right.\right. \\
& \left.\left.+2 x_{2} x_{2}^{\prime \prime}\right]\right\} .
\end{aligned}
$$

\section{Modes of a Resonator with Retroreflective Roof and Spherical Mirrors}

The integral equation Eq. (11) has been solved in the limit that the Fresnel number $a^{2} /(L \lambda)$ approaches infinity, where $2 a$ is the width of the spherical mirror and the confinement criterion $0<g_{2}<1$ is assumed to be satisfied. The solution of Eq. (11) in this case can be written in the form 


$$
E_{y}^{(2)}\left(y_{2}\right)=\phi_{n}\left(y_{2} / w_{2}\right),
$$

where $\phi_{n}$ is the normalized Hermite-Gaussian function. ${ }^{8}$

$$
\begin{aligned}
\phi_{n}\left(y_{2} / w_{2}\right) & =N_{n}^{(2)} H_{n}\left(\sqrt{2} y_{2} / w_{\dot{2}}\right) \exp \left(-y_{2}^{2} / w_{2}^{2}\right), \\
N_{n}^{(2)} & =\left(\sqrt{2} / w_{2}\right)^{1 / 2}\left(2^{n} n ! \sqrt{\pi}\right)^{-1 / 2}, \\
w_{2} & =(\pi / \lambda L)^{-1 / 2}\left[g_{2}\left(1-g_{2}\right)\right]^{-1 / 4} .
\end{aligned}
$$

Here $H_{n}$ is a Hermite polynomial of order $n, N_{n}^{(2)}$ is a normalization factor, and $w_{2}$ is the beam spot size on the spherical mirror. The corresponding eigenvalue can be expressed as

$$
\gamma_{y n}=\sigma_{n},
$$

where

$$
\begin{aligned}
\sigma_{n}= & \exp \left[-i k L+i(n+1 / 2)\left(\pi / 2+\tan ^{-1}\right.\right. \\
& \left.\left.\left\{\left(1-2 g_{2}\right)\left[1-\left(1-2 g_{2}\right)^{2}\right]^{-1 / 2}\right\}\right)\right] .
\end{aligned}
$$

To solve integral equation Eq. (14), part of the kernel can be expanded in terms of the products of eigenfunctions in both variables according to

$$
\begin{aligned}
(2 \lambda L)^{-1} \exp \{-i \pi / 4-i k L-i k & {\left.\left[\left(2 g_{2}-1\right)\left(x_{2}^{2}+x_{2}^{\prime 2}\right)-2 x_{2} x_{2}^{\prime \prime}\right] / 4 L\right\} } \\
& =\sum_{m} \sigma_{m} \phi_{m}\left(x_{2} / w_{2}\right) \phi_{m}\left(x_{2}^{*} / w_{2}\right) .
\end{aligned}
$$

Then Eq. (14) can be expressed in the form

$$
\gamma_{x} E_{x}^{(2)}\left(x_{2}\right)=\sum \sigma_{m} C_{m} \phi_{m}\left(x_{2} / w_{2}\right)
$$

where

$$
\begin{aligned}
C_{m} & =\int_{-\infty}^{\infty} d x_{2} E^{(2)}\left(-x_{2}^{\prime \prime}\right) \phi_{m}\left(x_{2}^{\prime \prime} / w_{2}\right) \\
& =\int_{-\infty}^{\infty} d x_{2} E^{(2)}\left(x_{2}^{\prime \prime}\right) \phi_{m}\left(-x_{2}^{\prime \prime} / w_{2}\right) .
\end{aligned}
$$

Multiplying both sides of Eq. (22) by $\phi_{n}\left(-x_{2} / w_{2}\right)$ and integrating, one obtains

$$
\begin{aligned}
E_{x m}^{(2)}\left(x_{2}\right) & =\phi_{m}\left(x_{2} / w_{2}\right), \\
\gamma_{x} & =(-1)^{m} \sigma_{m},
\end{aligned}
$$

where we have employed the orthogonality relation

$$
\int_{-\infty}^{\infty} \phi_{m}\left(x_{2} / w_{2}\right) \phi_{n}\left(-x_{2} / w_{2}\right) d x_{2}=(-1)^{m} \delta_{m n} .
$$

It is interesting that even for large inclination angles of the roof $\theta$, the field distribution can still be expressed in terms of an ordinary Hermite-Gaussian function, and the propagation constant $\gamma_{x m}$ is the same as for a common spherical resonator except for a multiplier $(-1)^{m}$. This multiplier means that the odd symmetrical modes have an additional phase shift of $\pi$. These results are consequences of the retroreflecting property of the right-angle roof mirror. The resonant frequency $\nu_{m n q}$ can be found from Eqs. (13), (19), and (25):

$$
\nu_{m n q}=(c / 2 L)\left[q-m / 2+(m+n+1)(\pi)^{-1} \cos ^{-1} g_{2}{ }^{1 / 2}\right] .
$$

After the field distribution on the spherical mirror has been determined the field variation in the $x$ direction on the imaginary plane $Q_{1} Q_{1}^{\prime}$ of the retroreflecting roof mirror can be derived by Fresnel integration

$$
E_{x m}^{(1)}=\chi_{m}^{\prime}\left(1-x_{1} \tan \theta / L\right) \exp \left(-i k x_{1} \tan \theta\right) \phi_{m}^{(1)}\left(x_{1} / w_{1}\right),
$$

where

$$
\begin{aligned}
\chi_{m}^{\prime}= & \exp \left(-i k L / 2+i(m+1 / 2)\left\{\pi / 2-\tan ^{-1}\right.\right. \\
& {\left.\left.\left[g_{2} /\left(1-g_{2}\right)\right]^{1 / 2}\right\}\right), } \\
\Phi_{m}^{(1)}\left(x_{1} / w_{1}\right)= & N_{m}^{(1)} H_{m}\left(\sqrt{2} x_{1} / w_{1}\right) \exp \left(-x_{1}^{2} / w_{1}^{2}\right), \\
N_{m}^{(1)}= & \left(\sqrt{2} / w_{1}\right)^{1 / 2}\left(2^{m} m ! \pi^{1 / 2}\right)^{-1 / 2}, \\
w_{1}= & (\pi / \lambda L)^{-1 / 2}\left[g_{2}^{-1}\left(1-g_{2}\right)\right]^{-1 / 4} .
\end{aligned}
$$

Here the phase factor $\exp \left(-i k x_{1} \tan \theta\right)$ comes from the inclination of the roof, and $\left(1-x_{1} \tan \theta / L\right)$ comes from the denominator in the integrand: Thus, the field distribution on the surface $Q_{1} Q_{1}^{\prime}$ is also very similar to that on the plane mirror of a conventional resonator.

\section{Beam Matrix for a Retroreflecting Roof}

Consider that an arbitrary Hermite-Gaussian beam expressed by $F_{m}\left(x_{2}\right)$ at $z$ propagates a distance $z$ and impinges on the tilted retroreflecting roof in such a way that a component ray travels through points $P_{1}^{\prime}$ and $A_{1}^{\prime}$ to $Q_{1}^{\prime}$ (see Fig. 1). Then the field variations in the $x$ direction on the surface of $Q_{1} Q_{1}^{\prime}$ can be derived by Fresnel-Kirchhoff integration:

$$
\begin{aligned}
F_{m}^{(1)}\left(x_{1}\right)= & (i / \lambda z)^{1 / 2}\left(1-x_{1} \tan \phi / z\right) \exp \left(-i k z / 2+i k x_{1} \tan \theta\right) \\
& \cdot \int d x_{2} F_{m}\left(x_{2}\right) \exp \left[-i k\left(x_{1}^{2}+x_{2}^{2}+2 x_{1} x_{2}\right) /(2 z)\right],
\end{aligned}
$$

where we have assumed that the optical axis of this beam passes through the apex of the roof. After reflection the beam propagates a distance $z$ to its starting point. The field variation in the $x$ direction can be found as follows:

$$
\begin{aligned}
F_{m}^{(3)}\left(x_{2}\right)= & (i / \lambda z)^{1 / 2} \int d x_{1} F_{m}^{(1)}\left(x_{1}\right)\left(1+x_{1} \tan \theta / z\right)^{-1} \\
& \cdot \exp \left[-i k z / 2-i k x_{1} \tan \theta-i k\left(x_{1}^{2}+x_{2}^{2}-2 x_{1} x_{2}\right) / 2 z\right] .
\end{aligned}
$$

Substituting Eq. (33) into Eq. (34) and using the same procedure as in Sec. IV, one gets

$$
\begin{aligned}
F_{m}^{(3)}\left(x_{2}\right)= & (-1)^{m}(2 \lambda z)^{-1 / 2} \exp (-i k z) \\
& \cdot \int_{-\infty}^{\infty} d x_{2}^{\prime} F_{m}\left(x_{2}^{\prime}\right) \exp \left[-i k\left(x_{2}^{2}+x_{2}^{\prime 2}-2 x_{2} x_{2}^{\prime}\right) /(4 z)\right] .
\end{aligned}
$$

On the other hand, we may also imagine that the same Hermite-Gaussian beam $F_{m}\left(x_{2}\right)$ simply travels a distance $2 z$. The field variation in the $x$ direction then can be written as

$$
\begin{aligned}
F_{m}^{\prime}\left(x_{3}\right)= & (2 \lambda z)^{-1 / 2} \exp (-i k z) \int_{-\infty}^{\infty} d x_{2}^{\prime} F_{m}\left(x_{2}^{\prime}\right) \\
& \cdot \exp \left[-i k\left(x_{2}^{2}+x_{3}^{2}-2 x_{2} x_{3}\right) /(4 z)\right] .
\end{aligned}
$$

Comparing Eq. (36) with Eq. (35) one finds that they are almost the same apart from an additional constant factor $(-1)^{m}$ in Eq. (35). Obviously this additional factor does not affect the spot size or the phase of the beam except for an additional phase shift of $\pi$. Using this information, together with Kogelnik's $A B C D$ transformation, ${ }^{9}$ it follows that the amplitude and phase distribution for the retroreflecting roof mirror can be characterized by the transformation matrix 


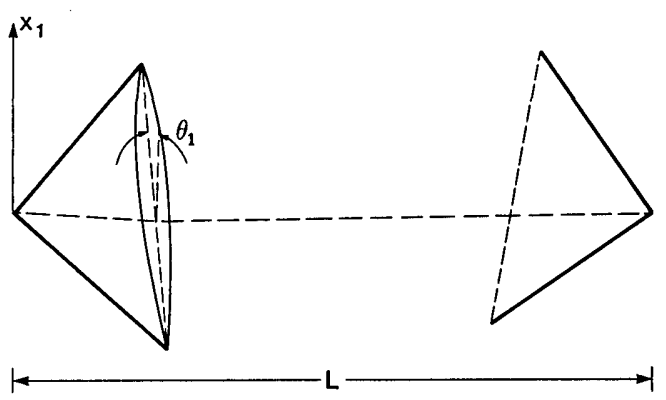

Fig. 2. Low loss resonator which does not need fine angular alignment.

$$
\left(\begin{array}{ll}
A & B \\
C & D
\end{array}\right)=\left(\begin{array}{cc}
-1 & 0 \\
0 & -1
\end{array}\right)
$$

When substituted into the appropriate phase transformation ${ }^{10,11}$ this matrix leads to the $(-1)^{m}$ factor noted above, and when applied as a ray matrix Eq. (37) also gives the expected transformation of position and slope. Thus this matrix can be used to calculate the modes of a complicated resonator which contains a retroreflecting roof.

As an example, consider a resonator consisting of two retroreflecting roofs and a thin cylindrical lens as shown in Fig. 2. The optical axis passes through the apexes of both roofs, and if the lens is rigidly attached to a reflector as suggested in the figure, the axis would be slightly bent at the lens. The cylindrical lens has a long focal length $f, f \gg w$ ( $w$ is the resulting spot size), and thus the beam matrix for a round trip starting just before the right-hand mirror can be calculated as

$$
\begin{aligned}
\left(\begin{array}{ll}
A & B \\
C & D
\end{array}\right)= & \left(\begin{array}{ll}
1 & L \\
0 & 1
\end{array}\right)\left(\begin{array}{ll}
1 & 0 \\
-\frac{\cos \theta}{f} & 1
\end{array}\right)\left(\begin{array}{rr}
-1 & 0 \\
0 & -1
\end{array}\right)\left(\begin{array}{ll}
1 & 0 \\
-\frac{\cos \theta}{f} & 1
\end{array}\right) \\
& \times\left(\begin{array}{ll}
1 & L \\
0 & 1
\end{array}\right)\left(\begin{array}{rr}
-1 & 0 \\
0 & -1
\end{array}\right), \\
= & \left(\begin{array}{ll}
1 & L \\
0 & 1
\end{array}\right)\left(\begin{array}{ll}
1 & 0 \\
-2 \frac{\cos \theta}{f} & 1
\end{array}\right)\left(\begin{array}{ll}
1 & L \\
0 & 1
\end{array}\right),
\end{aligned}
$$

where the $\cos \theta$ factor is the first correction that occurs whenever a lens is tilted. From this equation, one finds that the resonator is equivalent to an ordinary resonator with a plane mirror and a cylindrical mirror with radius of curvature

$$
R=f / \cos \theta \text {. }
$$

This rèsonator has some obvious advantages. First, the roofs do not need alignment in the $x$ direction except perhaps to keep the optical axis in line with the amplifying medium. Second, Eq. (39) suggests that in this configuration the effective radius of curvature $R$ may be changed by rotation of the mirror. It would, of course, also be possible to place a second cylindrical lens at the right-hand end of the cavity, and the desired output coupling could be obtained with suitable mirror coatings.

\section{Conclusion}

We have solved the self-consistent Fresnel-Kirchhoff integral equation for the field distribution of the resonant modes of a resonator with a tilted retroreflecting roof mirror and a spherical mirror with infinite Fresnel numbers. Even when the inclination angle of the roof mirror is quite large, low loss modes still exist, and the amplitude distributions of these modes can be expressed in terms of familiar Hermite-Gaussian functions. In hindsight, such modes might have been expected from the analogy of a retroreflecting mirror and an aligned flat mirror. The phase of the modes is, however, different from the phase in a conventional laser resonator, and hence the mode frequencies are different too.

The beam matrix for the retroreflecting roof mirror has also been discussed, and it may be written as simply minus one times the identity matrix. A new type of resonator has been proposed, and the unusual feature of this resonator is that it does not need fine angular alignment. This resonator might find applications in adverse environments where maintenance of laser alignment would otherwise be difficult. We are presently investigating the extension of these results to other types of retroreflecting laser systems.

Guosheng Zhou is a visiting scholar at UCLA from the Department of Physics of Shanxi University, China.

\section{Appendix}

The diagram used for calculation of path length differences is represented in Fig. 3. Two triangles $B O B_{1}$ and $B_{1} O B_{2}$ are congruent to the roof triangle $A O B$. The line $Q_{1} Q_{1}^{\prime}$ passes through the apex of the roof and is parallel to the roof surface $A B$. This line intersects the line $P_{1}^{\prime} S$ at $Q_{1}^{\prime}$ and intersects the line $P_{1} A_{1} Q_{1}$ at $Q_{1}$. An incident ray from $P_{1}^{\prime}$ experiences two reflections at $A_{1}^{\prime}$ and $A_{1}$ and goes to $P_{1}$ at the surface of the roof mirror. By simple geometry one can find that the ray $P_{1}^{\prime} A_{1}^{\prime} A_{1} P_{1}$ is equivalent to the ray $P_{1}^{\prime} Q_{1}^{\prime} S$ except that these rays propagate in different directions. Therefore the path length difference $\Delta=P_{1}^{\prime} A_{1}^{\prime}+A_{1}^{\prime} A_{1}+A_{1} P_{1}$ is equal to $P_{1}^{\prime} S$, which is given by

$$
\Delta=P_{1}^{\prime} S=d / \cos (\theta-\beta)
$$

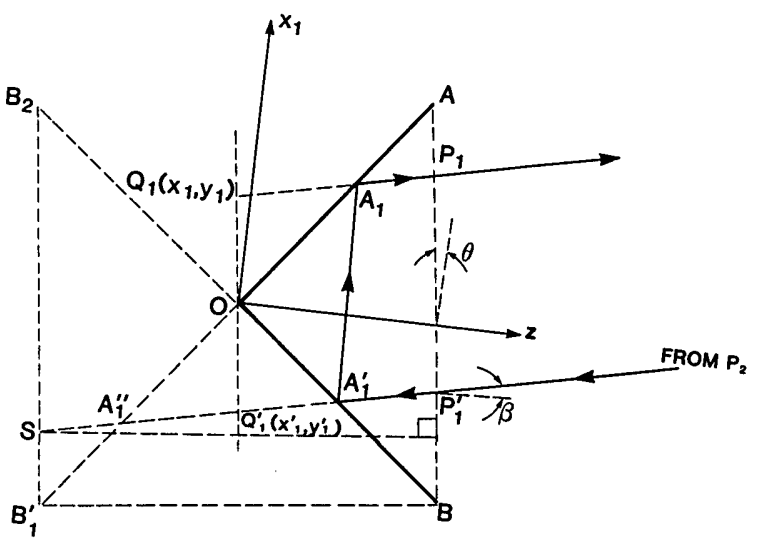

Fig. 3. Schematic representation for calculating path length differences. 
where $\theta$ is the angle between the $x$ axis and the roof surface, and $\beta$ is the angle between the incident ray and the $z$ axis. The latter angle may be approximately expressed as $\beta \simeq\left(x_{2}-x_{1}^{\prime}\right) / L$. Generally speaking, $\beta$ is of the order of $w / L \simeq 10^{-3} \mathrm{rad}$, while $\theta$ may be much larger. Therefore, $\cos (\theta-\beta)$ is approximately equal to $\cos \theta+\beta \sin \theta$, and

$$
\Delta \simeq d \sec \theta-d\left(x_{2}-x_{1}^{\prime}\right) \tan \theta \sec \theta / L .
$$

Obviously a ray from $P_{1}^{\prime}$ through $A_{1}^{\prime}$ and $A_{1}$ to $P_{1}$ can also be equivalent to a ray which propagates from $P_{1}^{\prime}$ through $A_{1}^{\prime}$ to $Q_{1}^{\prime}$, experiences a displacement $Q_{1}^{\prime} Q_{1}$, which does not contribute to any path length difference, and then travels from $Q_{1}$ through $A_{1}$ to $P_{1}$. Therefore, the plane $Q_{1} Q_{1}^{\prime}$ can be regarded as a reference plane for calculating the field.

Since $A_{1} O=O A_{1}^{\prime \prime}$ and $A_{1} Q_{1}$ is parallel to $Q_{1}^{\prime} A_{1}^{\prime \prime}$, the triangles $O Q_{1} A_{1}$ and $O Q_{1}^{\prime} A_{1}^{\prime \prime}$ are congruent. Therefore, $Q_{1} O=O Q_{1}^{\prime}$, or

$$
x_{1}=-x_{1}^{\prime} .
$$

The path length difference in Eq. (3) can be expressed in the form

$$
\begin{aligned}
\rho^{\prime}\left(x_{1}^{\prime}, x_{2}\right) & =L / 2+x_{1}^{\prime} \tan \theta+\left(x_{1}^{\prime 2}+g_{2} x_{2}^{2}-2 x_{1}^{\prime} x_{2}\right) / 2 L, \\
& =L / 2-x_{1} \tan \theta+\left(x_{1}^{2}+g_{2} x_{2}^{2}+2 x_{1} x_{2}\right) / 2 L,
\end{aligned}
$$

and this result is used in reducing Eqs. (8) and (9).

\section{References}

1. G. Toraldo di Francia, Appl. Opt. 4, 1267 (1965).

2. W. K. Kahn, Appl. Opt. 6, 865 (1967).

3. A. G. Fox and T. Li, Bell Syst. Tech. J. 40, 453 (1961).

4. P. F. Checcaci, A. Consortini, and A. Scheggi, Proc. IEEE, 54, 1329 (1966).

5. P. F. Checcaci, A. Consortini, and A. Scheggi, Appl. Opt. 65, 1567 (1966).

6. L. Bergstein, W. Kahn, and C. Shulman, Proc. IRE Eng. 50, 1833 (1962).

7. See Ref. 3, Eq. (22). The term $x_{1}^{\prime} \tan \theta$ in our Eq. (3) is due to rotation of the left-hand reference plane. Here we have assumed that $(\tan \theta)^{2} \ll 1$

8. P. O. Clark, Proc. IEEE 53, 36 (1965).

9. H. Kogelnik and T. Li, Appl. Opt. 5, 1550 (1966).

10. J. -P. Taché, Appl. Opt. 19, 4214 (1980), Eq. (23).

11. L. W. Casperson, Appl. Opt. 20, 2243 (1981), Eq. (10).

\section{Simple solar tracking device activates motor to focus collector}

A patent on a simple solar collector tracking apparatus has been awarded to the Department of Energy in the name of its inventor, Robert P. Stromberg of Sandia National Laboratories. Patent number is $4,274,394$.

The device, which activates a motor to drive a collector into focus, consists basically of two parallel bimetallic strips separated by a shade plate. The strips, which are about two inches by one-half inch and similar to those found in many thermostats, are attached at one end to a horizontal shaft. One strip is also anchored at the other end, but the end of the second strip is an electrical contact suspended midway between two fixed contacts.

\section{Equally exposed to sun}

When the two strips are equally exposed to the sun, the collector is in focus and the electrical circuit from the sensor is open. However, when the strips are unequally illuminated, indicating the collector is not in focus, the circuit is closed by the flexing action of one of the strips. Closure of the overhead contact drives the motor in one direction, while closure of the bottom contact drives the motor in the other direction.

The shade plate is sufficient for coarse tracking: for fine tracking, a shadow bar is located along the collector axis about 12 inches above the centerline of the two strips.

Thermal lag in the strips permits the collector to be driven squarely into focus before the circuit is broken, stopping the tracking motion. Tracking also stops during periods of cloudiness because insufficient heat is created to flex the strips.

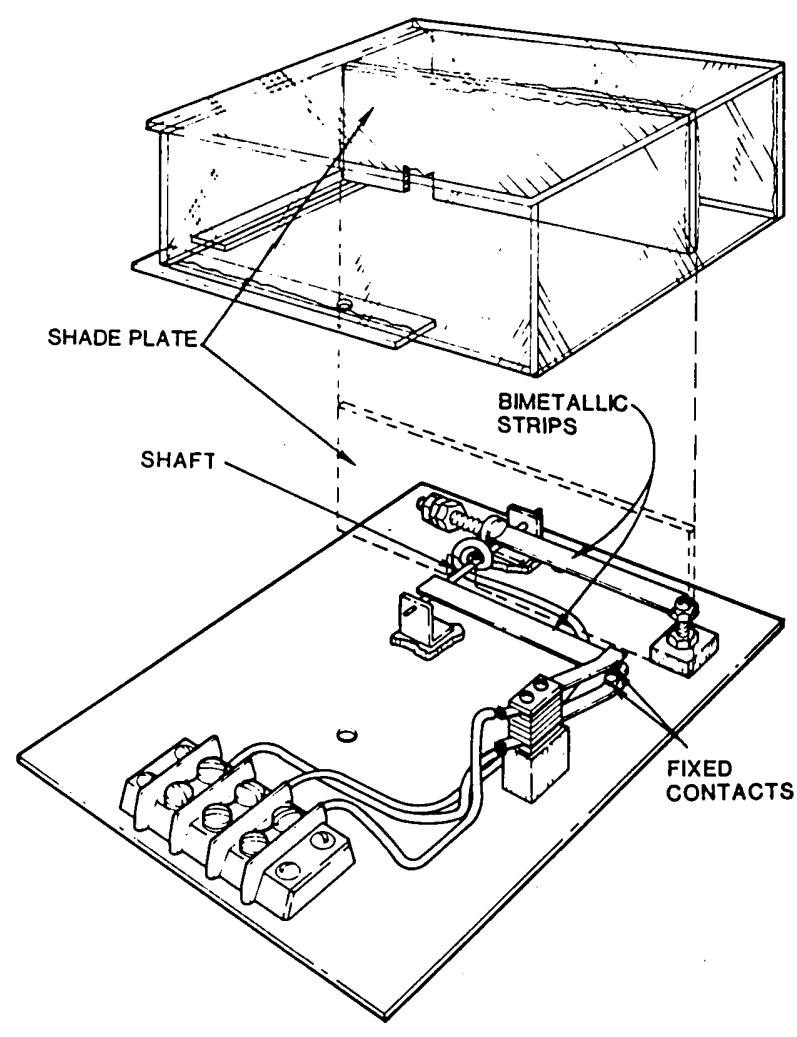

SOLAR COLLECTOR TRACKING DEVICE 